\title{
A NOTE ON THE PRODUCT OF MEROMORPHIC FUNCTIONS AND ITS DERIVATIVES
}

\author{
Kit-Wing YU
}

\begin{abstract}
It is shown that if $f$ is an even or odd transcendental meromorphic function and if $c$ is any even meromorphic function which does not vanish identically and satisfies $T(r, c)=o(T(r, f))$ as $r \rightarrow+\infty$, then $f f^{\prime}-c$ has infinitely many zeros.
\end{abstract}

\section{Introduction and our main results}

In 1959, W. K. Hayman [5] proved that

THEOREM A. If $n$ is an integer greater than or equal to 3 and $f$ is a transcendental meromorphic function, then $f^{n} f^{\prime}$ takes every non-zero complex number infinitely many times.

Later, he conjectured [6] that this remains valid for the cases $n=1,2$. In 1979, E. Mues [7] proved the case $n=2$ and the conjecture was proven by A. Eremenko and W. Bergweiler [2] in 1995 and independently by H. H. Chen and M. L. Fang [3].

In 1994, Yik-Man Chiang asked W. Bergweiler whether $f f^{\prime}-c$ has infinitely many zeros if $f$ is a transcendental meromorphic function and if $c$ is a meromorphic function which does not vanish identically and satisfies $T(r, c)=o(T(r, f))$ as $r \rightarrow+\infty$. In [8], Q. D. Zhang studied the value distribution of $\varphi(z) f(z) f^{\prime}(z)$ and obtained the following theorem.

THEOREM B. If $f$ is a transcendental meromorphic function and $\varphi$ is a nonzero meromorphic function such that $T(r, \varphi)=S(r, f)$ as $r \rightarrow+\infty$, then

$$
T(r, f)<\frac{9}{2} \bar{N}(r, f)+\frac{9}{2} \bar{N}\left(r, \frac{1}{\varphi f f^{\prime}-1}\right)+S(r, f) .
$$

By this, we have

2000 Mathematics Subject Classfication: Prelimary 30D35.

Key words: derivatives, meromorphic functions, zeros.

Received July 21, 2000; revised January 5, 2001. 
COROLlary A. If $\delta(\infty, f)>7 / 9$, then $f f^{\prime}-c$ has infinitely many zeros.

In 1995, W. Bergweiler and A. Eremenko [2] used some results from iteration theory to show that $f f^{\prime}-c$ has infinitely many zeros if $c$ is a non-zero constant. Later, W. Bergweiler [1] answered this question affirmatively in the case that $f$ is of finite order and $c$ is a polynomial. Actually, he showed that

THEOREM C. If $f$ is a transcendental meromorphic function of finite order and $c$ is a polynomial, then $f f^{\prime}-c$ has infinitely many zeros.

In the following discussion, we assume that $f$ is a transcendental meromorphic function. From Corollary $\mathrm{A}$, we can further assume that $\delta(\infty, f) \leq 7 / 9$ and we shall show the following result.

THEOREM. Let $f$ be a transcendental meromorphic function and $c$ be a meromorphic function which does not vanish identically and satisfies $T(r, c)=$ $o(T(r, f))$ as $r \rightarrow+\infty$. Then $f f^{\prime}-c$ or $f f^{\prime}+c$ has infinitely many zeros.

COROllary. Suppose that $f$ is an even or odd transcendental meromorphic function and $c$ is an even meromorphic function. Suppose further that there exists a sequence $\left\{z_{1},-z_{1}, z_{2},-z_{2}, \ldots,\right\}$ which are zeros of $\varphi f f^{\prime}+1$ but not zeros or poles of $f$. Then $f f^{\prime}-c$ has infinitely many zeros.

Here, we assume that the readers are familiar with the basic concepts of the Nevanlinna value distribution theory and the notations $m(r, f), N(r, f), \bar{N}(r, f)$, $T(r, f)$ and etc., see e.g., [4].

\section{A lemma}

Lemma. Suppose that $f$ is a non-constant meromorphic function and that $\varphi$ is a non-vanishing meromorphic function such that $T(r, \varphi)=o(T(r, f))$ as $r \rightarrow+\infty$. Then for any finite non-zero distinct complex numbers $a$ and $b$ and any positive integer $k$ such that $\varphi f^{(k)} \not \equiv$ constant, we have

$$
\begin{aligned}
T(r, f)< & N\left(r, \frac{1}{f}\right)+N\left(r, \frac{1}{\varphi f^{(k)}-a}\right)+N\left(r, \frac{1}{\varphi f^{(k)}-b}\right) \\
& -N(r, f)-N\left(r, \frac{1}{\left(\varphi f^{(k)}\right)^{\prime}}\right)+S(r, f)
\end{aligned}
$$

as $r \rightarrow+\infty$.

Proof. First of all, we have

$$
m\left(r, \frac{1}{\varphi f}\right) \leq m\left(r, \frac{1}{\varphi f^{(k)}}\right)+m\left(r, \frac{f^{(k)}}{f}\right)+O(1) .
$$


From

$$
\begin{gathered}
m\left(r, \frac{1}{\varphi f}\right)=T(r, \varphi f)-N\left(r, \frac{1}{\varphi f}\right)+O(1), \\
m\left(r, \frac{1}{\varphi f^{(k)}}\right)=T\left(r, \varphi f^{(k)}\right)-N\left(r, \frac{1}{\varphi f^{(k)}}\right)+O(1),
\end{gathered}
$$

and (1), we have

$$
\begin{aligned}
T(r, \varphi f) \leq & N\left(r, \frac{1}{\varphi f}\right)+T\left(r, \varphi f^{(k)}\right)-N\left(r, \frac{1}{\varphi f^{(k)}}\right) \\
& +m\left(r, \frac{f^{(k)}}{f}\right)+O(1) .
\end{aligned}
$$

By the second fundamental theorem,

$$
\begin{aligned}
T\left(r, \varphi f^{(k)}\right)< & N\left(r, \frac{1}{\varphi f^{(k)}}\right)+N\left(r, \frac{1}{\varphi f^{(k)}-a}\right)+N\left(r, \frac{1}{\varphi f^{(k)}-b}\right) \\
& -N_{1}(r)+S\left(r, \varphi f^{(k)}\right)
\end{aligned}
$$

as $r \rightarrow+\infty$, where, as usual, $N_{1}(r)$ is defined as

$$
N_{1}(r)=2 N\left(r, \varphi f^{(k)}\right)-N\left(r,\left(\varphi f^{(k)}\right)^{\prime}\right)+N\left(r, \frac{1}{\left(\varphi f^{(k)}\right)^{\prime}}\right) .
$$

Let $z_{0}$ be a pole of order $p \geq 1$ of $f$. Then $f^{(k)}$ and $f^{(k+1)}$ have a pole of order $k+p$ and $k+p+1$ at $z_{0}$ respectively. Thus $2(k+p)-(k+p+1)=$ $k+p-1 \geq p$ and

$$
N_{1}(r) \geq N(r, f)+N\left(r, \frac{1}{\left(\varphi f^{(k)}\right)^{\prime}}\right)+S(r, f)
$$

It is clear that $S\left(r, f^{(k)}\right)=S(r, f)$ and $m\left(r, \frac{f^{(k)}}{f}\right)=S(r, f)$. Thus by (2), (3)
(4), and (4),

$$
\begin{aligned}
T(r, \varphi f)< & N\left(r, \frac{1}{\varphi f}\right)+N\left(r, \frac{1}{\varphi f^{(k)}-a}\right)+N\left(r, \frac{1}{\varphi f^{(k)}-b}\right) \\
& -N(r, f)-N\left(r, \frac{1}{\left(\varphi f^{(k)}\right)^{\prime}}\right)+S(r, f)
\end{aligned}
$$

as $r \rightarrow+\infty$. Since $T(r, \varphi)=o(T(r, f))$ as $r \rightarrow+\infty$, we have the desired result. 


\section{Proofs of the theorem and the corollary}

Proof of the theorem. Let $\varphi=1 / c, F=(1 / 2) f^{2}, k=1, a=1$ and $b=-1$. Then by the above lemma, we have

$$
\begin{aligned}
2 T(r, f)< & 2 N\left(r, \frac{1}{f}\right)+N\left(r, \frac{1}{\varphi f f^{\prime}-1}\right)+N\left(r, \frac{1}{\varphi f f^{\prime}+1}\right) \\
& -2 N(r, f)-N\left(r, \frac{1}{\left(\varphi f f^{\prime}\right)^{\prime}}\right)+S(r, f)
\end{aligned}
$$

as $r \rightarrow+\infty$.

By the assumption that $\delta(\infty, f) \leq 7 / 9$, we have

$$
\frac{N\left(r, \frac{1}{\varphi f f^{\prime}-1}\right)+N\left(r, \frac{1}{\varphi f f^{\prime}+1}\right)}{2 T(r, f)}>0
$$

as $r \rightarrow+\infty$. Hence, the result of the theorem follows.

Proof of the corollary. If

$$
\frac{N\left(r, \frac{1}{\varphi f f^{\prime}-1}\right)}{2 T(r, f)}>0
$$

as $r \rightarrow+\infty$ outside a set of finite linear measure, then $\varphi f f^{\prime}-1$ has infinitely many zeros and thus $f f^{\prime}-c$ has infinitely many zeros.

Let $z_{0} \neq 0$ be a zero of $\varphi f f^{\prime}+1$. Since $f$ is even or odd, $f^{\prime}$ is odd or even. Therefore, $f f^{\prime}$ is an odd function. Now we have $\varphi\left(-z_{0}\right) f\left(-z_{0}\right) f^{\prime}\left(-z_{0}\right)-1=$ $-\varphi\left(z_{0}\right) f\left(z_{0}\right) f^{\prime}\left(z_{0}\right)-1=0$ and thus $-z_{0}$ is a zero of $\varphi f f^{\prime}-1$. Hence, the desired result follows.

\section{Remarks}

1. In [8], Q. D. Zhang showed that

$$
2 T(r, f)<\bar{N}(r, f)+2 \bar{N}\left(r, \frac{1}{f}\right)+\bar{N}\left(r, \frac{1}{\varphi f f^{\prime}-1}\right)+S(r, f) .
$$

From this, it is immedicate that if $\delta(0, f)+(1 / 2) \delta(\infty, f)>1 / 2$, then $f f^{\prime}-c$ has infinitely many zeros, where $c=1 / \varphi$.

2. In [9], Z. F. Zhang and G. D. Song showed that if $a(z) \not \equiv 0$ and $T(r, a)=S(r, f)$ as $r \rightarrow+\infty, n, k$ are positive integers, where $n \geq 2$, then $f\left(f^{(k)}\right)^{n}-a(z)$ has infinitely many zeros. 
Acknowledgement. The author wishes to thank the referee for many valuable comments.

\section{REFERENCES}

[1] W. Bergweiler, On the product of a meromorphic function and its derivative, Bull. Hong Kong Math. Soc., 1 (1996), 97-101.

[2] W. Bergweiler and A. Eremenko, On the singularities of the inverse of a meromorphic function of finite order, Rev. Mat. Iberoamericana, 11 (1995), 355-373.

[ 3 ] H. H. Chen And M. L. FAng, On the value distribution of $f^{n} f^{\prime}$, Science in China Ser. A, 25 (1995), 121-127.

[4] W. K. Hayman, Meromorphic Functions, Oxford Math. Monogr., Clarendon Press, Oxford, 1964.

[5] W. K. Hayman, Picard values of meromorphic functions and their derivatives, Ann. of Math., 70 (1959), 9-42.

[6] W. K. Hayman, Research Problems in Function Theory, The Athlone Press, London, 1967.

[ 7 ] E. Mues, Über ein Problem von Hayman, Math. Z., 164 (1979), 239-259.

[ 8 ] Q. D. Zhang, On the value distribution of $\varphi(z) f(z) f^{\prime}(z)$, Acta Math. Sinica, 37 (1994), $91-$ 97 (in Chinese).

[9] Z. F. Zhang And G. D. Song, On the zeros of $f\left(f^{(k)}\right)^{n}-a(z)$, Chinese Ann. Math. Ser. A, 19 (1998), 275-282.

Department of Mathematics

The Hong Kong University of Science \& Technology

Clear Water Bay, Kowloon, Hong Kong

e-mail: makwing@ust.hk

(CURRENT ADDRESS)

Rm. 205, Kwai Shun Hse., Kwai Fong Est.,

K.C., Hong Kong

e-mail: kitwing@hotmail.com 